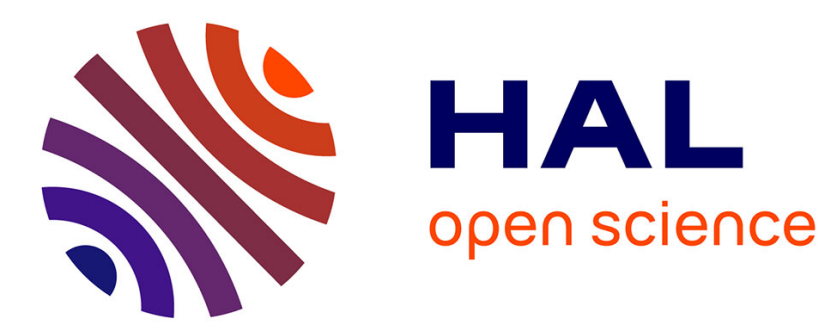

\title{
Direct finite element computation of non-linear modal coupling coefficients for reduced-order shell models
}

\author{
Cyril Touzé, Marina Vidrascu, Dominique Chapelle
}

\section{To cite this version:}

Cyril Touzé, Marina Vidrascu, Dominique Chapelle. Direct finite element computation of non-linear modal coupling coefficients for reduced-order shell models. Computational Mechanics, 2014, 54, pp.567-580. 10.1007/s00466-014-1006-4 . hal-00955582

\section{HAL Id: hal-00955582 \\ https://hal.inria.fr/hal-00955582}

Submitted on 4 Mar 2014

HAL is a multi-disciplinary open access archive for the deposit and dissemination of scientific research documents, whether they are published or not. The documents may come from teaching and research institutions in France or abroad, or from public or private research centers.
L'archive ouverte pluridisciplinaire HAL, est destinée au dépôt et à la diffusion de documents scientifiques de niveau recherche, publiés ou non, émanant des établissements d'enseignement et de recherche français ou étrangers, des laboratoires publics ou privés. 


\title{
Direct finite element computation of non-linear modal coupling coefficients for reduced-order shell models
}

\author{
C. Touzé · M. Vidrascu · D. Chapelle
}

Computational Mechanics, DOI:10.1007/s00466-014-1006-4

\begin{abstract}
We propose a direct method for computing modal coupling coefficients - due to geometrically nonlinear effects - for thin shells vibrating at large amplitude and discretized by a finite element (FE) procedure. These coupling coefficients arise when considering a discrete expansion of the unknown displacement onto the eigenmodes of the linear operator. The evolution problem is thus projected onto the eigenmodes basis and expressed as an assembly of oscillators with quadratic and cubic nonlinearities. The nonlinear coupling coefficients are directly derived from the finite element formulation, with specificities pertaining to the shell elements considered, namely, here elements of the "Mixed Interpolation of Tensorial Components" family (MITC). Therefore, the computation of coupling coefficients, combined with an adequate selection of the significant eigenmodes, allows the derivation of effective reduced-order models for computing - with a continuation procedure - the stable and unstable vibratory states of any vibrating shell, up to large amplitudes. The procedure is illustrated on a hyperbolic paraboloid panel. Bifurcation diagrams in free and forced vibrations are obtained. Comparisons with direct time simulations of the full FE model are given. Finally, the computed coeffi-
\end{abstract}

C. Touzé

Unité de Mécanique (UME),

ENSTA-ParisTech, 828 Boulevard des maréchaux,

91762 Palaiseau Cedex, France

Tel.: +33-1-69-31-97-34

E-mail: cyril.touze@ensta-paristech.fr

M. Vidrascu

Inria/Reo and LJLL UMR 7958 UPMC,

Rocquencourt, B.P. 105, 78153 Le Chesnay, France

E-mail: marina.vidrascu@inria.fr

D. Chapelle

Inria/M $\Xi D I S I M, 1$ rue Honoré d'Estienne d'Orves,

Campus de l'Ecole Polytechnique, 91120 Palaiseau, France

E-mail: dominique.chapelle@inria.fr 
cients are used for a maximal reduction based on asymptotic nonlinear normal modes (NNMs), and we find that the most important part of the dynamics can be predicted with a single oscillator equation.

Keywords geometric nonlinearity · finite elements · MITC elements · stiffness evaluation $\cdot$ bifurcation diagram $\cdot$ reduced-order models

\section{Introduction}

Thin shells vibrating at large amplitude can exhibit complex dynamics. These geometrically nonlinear behaviors occur as soon as the vibration amplitude is of the order of the thickness, and may induce various nonlinear effects such as jumps, instabilities, quasi-periodic or chaotic vibrations $[1,2]$. In turn, this may lead to undesirable vibration patterns that can have detrimental effects on the usual predicted behavior of numerous engineering systems, such as sudden increase in vibration amplitudes, fatigue of components, etc.. In order to have a significant understanding of the possible nonlinear behaviors of a given structure, the computation of a complete bifurcation diagram is key, as it gives access to all the solution branches (stable and unstable) under variations of some selected control parameters. For that purpose, direct numerical integration generally appears as cumbersome and inappropriate, as unstable states are not accessible to the computation. Moreover, computing all the solution branches by successive runs, given variable initial conditions, is so time-consuming that the method is usually not considered.

In this context, reduced-order models (ROMs) are generally much better adapted. In conjunction with a numerical continuation method [3,4], or perturbation analytical methods [5], one is able to obtain complete bifurcation diagrams for free vibrations and forced responses of thin structures. In the last years, many applications have been pursued using this methodology, in order to compute frequency response curves of thin structures harmonically forced in the vicinity of one of its eigenmodes, see e.g. $[5,2,6-9]$. In most contributions, the Partial Differential Equations (PDEs) of motion for a given shell model - following e.g. von Kármán assumptions, or Donnell shallow-shell theory, see e.g. [10] - is discretized by using the eigenmodes of the linear operator, or a given ad-hoc functional basis that satisfies the boundary conditions. Applying a Galerkin procedure, the problem is then transformed into a dynamical system by conserving the important modes, for which continuation methods can thus be applied. Unfortunately, this strategy is restricted to simple geometries, for which ad-hoc functional bases made of simple - often analytical - functions can be constructed, ensuring convergence for a small number of modes. For a general shell geometry, finding such a specific discretization method is much more difficult, see e.g. [11] for a proposed approach based on so-called R-functions.

For complex geometries the most common framework consists in using the versatility of finite-elements (FE) procedures. However, at the time being, 
there exists no contribution on reduced-order models based on shell finite elements for predicting bifurcation diagrams by a continuation method. More precisely, first attempts toward this general objective can be found in [12] for beam-like structures, and in [13,14] for rectangular plates. The present paper aims at proposing a complete strategy specifically adapted to tackle the most general case of thin shells. The main difficulty resides in the computation of the ROM from the FE discretized shell. A simple strategy that is used in this contribution consists in utilizing the eigenmodes basis in the construction process of the ROM. The dynamical problem, expressed onto the linear eigenmodes, is represented by an assembly of oscillators with quadratic and cubic nonlinearities, arising from the geometrically nonlinear terms. Within that framework, numerous nonlinear coupling coefficients appear in the dynamical system, and one needs to evaluate these coefficients in order to build the ROM.

Indirect determination of these nonlinear coupling coefficients has already been proposed. Muravyov [15], then Mignolet and Soize [16] used a so-called STEP method (STiffness Evaluation Procedure) for that purpose. The idea is to prescribe in the structural model numerous selected static deformations, taken from one of the eigenmodes or a combination thereof. From the computation of the residual, and via algebraic manipulations, nonlinear coupling coefficients can be evaluated. A review of the computational schemes, as well as their applications to solve numerous engineering problems involving for example random vibrations, is given in [17]. The main advantage of the STEP method is that one can use any commercial finite element software, as there is no need to compute specific finite element quantities, and only standard computations with specific post-processing allow to derive the desired coefficients. The drawback of this indirect method is that numerous well-selected combinations of static deformations must be considered; moreover, the method requires prescribing an appropriate amplitude for the static deflections.

In this contribution, we propose and implement a direct method with application to FE shells discretized with general shell elements of the MITC family (Mixed Interpolation of Tensorial Components) [18]. First an analytical expression of the nonlinear coupling coefficients is derived from the internal potential energy. The FE procedure and implementation details are then given. Next, the method is applied to a clamped hyperbolic paraboloid panel. In this case, the detailed derivation of ROMs is explained, and bifurcation diagrams in free and forced vibrations are given.

\section{Direct computation of nonlinear stiffness}

This section gives the analytical and implementation details for the direct computation of the nonlinear coupling coefficients describing the geometrical nonlinearity of the shell. The linear modes basis is used to discretize the FE problem, and specificities related to the use of the chosen shell elements are then thoroughly explained so as to highlight the practical implementation of the calculation in a given shell FE code. 


\subsection{Formulation}

This section is devoted to the analytical expressions of the nonlinear coupling coefficients. Geometric nonlinearity is assumed, which means that the material has a linear elastic behavior, but the shell can undergo large amplitude motions. In this context, the nonlinearities can be directly derived from the variations of internal elastic energy $\delta W_{i n t}$, which read

$$
\delta W_{i n t}=\int_{\Omega} \underline{\underline{\Sigma}}: \delta \underline{\underline{e}},
$$

where $\underline{\underline{\underline{\Sigma}}}$ is the second Piola-Kirchhoff stress tensor and $\underline{\underline{e}}$ the Green-Lagrange strain tensor. The strain-displacement relationship is

$$
\underline{\underline{e}}=\frac{1}{2}\left(\underline{\underline{\nabla}}+\underline{\underline{\nabla}}^{t}+\underline{\underline{\nabla}}^{t} \cdot \underline{\underline{\nabla}}\right) \underline{y}
$$

where $\underline{y}$ stands for the displacement. For simplicity, let us denote by $\underline{\underline{\varepsilon}}$ the linear part of the strain-displacement relationship

$$
\underline{\underline{\varepsilon}}=\frac{1}{2}\left(\underline{\underline{\nabla}}+\underline{\underline{\nabla}}{ }^{t}\right) \underline{y}
$$

and $\underline{e}^{(2)}$ the quadratic part

$$
\underline{\underline{e}}^{(2)}=\frac{1}{2}\left(\underline{\underline{\nabla}}^{t} \underline{y} \cdot \underline{\underline{\nabla}} \underline{y}\right)
$$

A linear elastic and isotropic material is assumed, so that we have

$$
\underline{\underline{\underline{E}}}=\underline{\underline{\underline{H}}}: \underline{\underline{e}}
$$

where $\underline{\underline{H}}$ stands for the constitutive tensor associated with a Saint-VenantKirchhoff material, namely, Hooke's law.

Then we have

$$
\delta W_{i n t}=\int_{\Omega}\left(\underline{\underline{\varepsilon}}+\underline{\underline{e}}^{(2)}\right): \underline{\underline{\underline{\underline{H}}}}:\left(\delta \underline{\underline{\varepsilon}}+\delta \underline{\underline{e}}^{(2)}\right) .
$$

From this last equation, one can identify the linear, quadratic and cubic terms (as functions of the displacement), which are denoted respectively by $\delta W_{1}$, $\delta W_{2}$ et $\delta W_{3}$

$$
\begin{aligned}
& \delta W_{1}=\int_{\Omega} \underline{\underline{\varepsilon}}: \underline{\underline{\underline{\underline{H}}}}: \delta \underline{\underline{\varepsilon}}, \\
& \delta W_{2}=\int_{\Omega} \underline{\underline{e}}^{(2)}: \underline{\underline{\underline{H}}}: \delta \underline{\underline{\varepsilon}}+\underline{\underline{\varepsilon}}: \underline{\underline{\underline{H}}}: \delta \underline{\underline{e}}^{(2)}, \\
& \delta W_{3}=\int_{\Omega} \underline{\underline{e}}^{(2)}: \underline{\underline{\underline{H}}}: \delta \underline{\underline{e}}^{(2)} .
\end{aligned}
$$


Let us now assume that the linear eigenmodes of the system $\underline{\Phi}_{p}(\underline{x})$ are known. The unknown displacement $\underline{y}(\underline{x}, t)$ is expanded onto the eigenmodes $\underline{\Phi}_{p}(\underline{x})$ as

$$
\underline{y}(\underline{x}, t)=\sum_{p=1}^{\infty} X_{p}(t) \underline{\Phi}_{p}(\underline{x}) .
$$

Expression (10) is inserted into the equations of motion containing the internal elastic forces associated with (6) in weak form. A Galerkin projection where the test functions used are the eigenmodes $\underline{\Phi}_{p}(\underline{x})$ allows to derive a infinite set of ordinary differential equations in the unknowns $X_{p}(t)$ driving the dynamics of the problem [19]. In view of (6), this set of oscillator equations contains only quadratic and cubic nonlinearities in the displacement variable, and thus has the generic form:

$$
\ddot{X}_{p}+\omega_{p}^{2} X_{p}+\sum_{i, j=1}^{\infty} g_{i j}^{p} X_{i} X_{j}+\sum_{i, j, k=1}^{\infty} h_{i j k}^{p} X_{i} X_{j} X_{k}=f_{p}(t) .
$$

In this expression, $\omega_{p}$ stands for the radian eigenfrequency of the mode labelled $p$. Note that no linear coupling terms are present in-between the modal oscillator equations, as a property of the linear modes basis. The non-linear coefficients $g_{i j}^{p}$ and $h_{i j k}^{p}$ are directly obtained by substituting the expansion (10) in (8) and (9), respectively, and choosing $\delta y=\underline{\Phi}_{p}$ as a test function.

Other basis functions could have been used for the Galerkin projection, such as POD modes (Proper Orthogonal Decomposition, see for instance [20$22]$ ). The general methodology explained here then remains obviously applicable. However, we specifically select linear eigenmodes as a projection basis for the following two reasons:

- it allows for a decoupling between linear components,

- eigenmodes are easily computable in any standard FE code.

Nonlinear terms appear through the coupling coefficients denoted by $g_{i j}^{p}$ (quadratic) and $h_{i j k}^{p}$ (cubic). Their expressions are now derived from the general elastic energy.

We can write the following expression

$$
\underline{\underline{e}}^{(2)}=\frac{1}{2} \sum_{i=1}^{\infty} \sum_{j=1}^{\infty} X_{i} X_{j}\left(\underline{\underline{\nabla}} \underline{\Phi}_{i}\right)^{t} \cdot\left(\underline{\underline{\nabla}}_{\underline{\Phi}}\right)
$$

and the quadratic term $\delta W_{2}$ then reads for $\delta \underline{y}=\underline{\Phi}_{p}$

$$
\begin{aligned}
& \delta W_{2}=\frac{1}{4} \sum_{i, j}\left(\int_{\Omega}\left(\underline{\underline{\nabla}} \underline{\Phi}_{i}\right)^{t} \cdot\left(\underline{\underline{\nabla}} \underline{\Phi}_{j}\right): \underline{\underline{\underline{H}}}:\left(\underline{\underline{\nabla}} \underline{\Phi}_{p}+\underline{\underline{\nabla}}^{t} \underline{\Phi}_{p}\right)\right) X_{i} X_{j} \\
& +\left(\int_{\Omega}\left(\underline{\underline{\nabla}} \underline{\Phi}_{i}+\underline{\underline{\nabla}}^{t} \underline{\Phi}_{i}\right): \underline{\underline{\underline{\underline{H}}}}:\left(\underline{\underline{\nabla}}^{t} \underline{\Phi}_{p} \cdot \underline{\underline{\nabla}} \underline{\Phi}_{j}+\underline{\underline{\nabla}}^{t} \underline{\Phi}_{j} \cdot \underline{\underline{\nabla}} \underline{\Phi}_{p}\right)\right) X_{i} X_{j}
\end{aligned}
$$


From this expression the quadratic coupling term can be easily derived as

$$
\begin{aligned}
& g_{i j}^{p}=\frac{1}{4} \int_{\Omega} \underline{\underline{\nabla}}^{t} \underline{\Phi}_{i} \cdot\left(\underline{\underline{\nabla}} \underline{\Phi}_{j}\right): \underline{\underline{\underline{\underline{H}}}}:\left(\underline{\underline{\nabla}} \underline{\Phi}_{p}+\underline{\underline{\nabla}}^{t} \underline{\Phi}_{p}\right) \\
& +\left(\underline{\underline{\nabla}} \underline{\Phi}_{i}+\underline{\underline{\nabla}}^{t} \underline{\Phi}_{i}\right): \underline{\underline{\underline{\underline{H}}}}:\left(\underline{\underline{\nabla}}^{t} \underline{\Phi}_{p} \cdot \underline{\underline{\nabla}} \underline{\Phi}_{j}+\underline{\underline{\nabla}}^{t} \underline{\Phi}_{j} \cdot \underline{\underline{\nabla}} \underline{\Phi}_{p}\right) .
\end{aligned}
$$

Likewise, the cubic term is derived as

$$
h_{i j k}^{p}=\frac{1}{4} \int_{\Omega} \underline{\underline{\nabla}}^{t} \underline{\Phi}_{i} \cdot \underline{\underline{\nabla}} \underline{\Phi}_{j}: \underline{\underline{\underline{\underline{H}}}}:\left(\underline{\underline{\nabla}}^{t} \underline{\Phi}_{p} \cdot \underline{\underline{\nabla}} \underline{\Phi}_{k}+\underline{\underline{\nabla}}^{t} \underline{\Phi}_{k} \cdot \underline{\underline{\nabla}} \underline{\Phi}_{p}\right) .
$$

\subsection{Finite element discretization}

The above derivation of non-linear coupling coefficients was performed in the abstract setting of a continuous model. In practice, of course, a discrete model is used, and in this paper we consider shell finite elements. This will have an impact on the expression of the non-linear coefficients, as the internal deformation energy $\delta W_{\text {int }}$ from which they are inferred needs to be modified in the shell finite element formulation, for reasons that will be made clear below.

The shell geometry is discretized using a mesh with nodes located on the mid-surface. More precisely, the 3D position within each element is given by

$$
\underline{x}=\sum_{i=1}^{N_{\text {nodes }}} \lambda_{i}(r, s)\left(\underline{x}^{(i)}+z \frac{t^{(i)}}{2} \underline{a}_{3}^{(i)}\right),
$$

where $\underline{x}^{(i)}, \underline{a}_{3}^{(i)}$ and $t^{(i)}$ respectively denote the position, normal vector, and thickness value associated with node $i$, while $(r, s, z)$ are the local coordinates within the element, and $\lambda_{i}$ the $2 \mathrm{D}$ shape functions considered. In our case we will use 4-node quadrilateral elements, with bilinear shape functions, and all local coordinates lie in $[-1,1]$.

We consider general shell elements [23,18], hence discrete displacements are defined according to an isoparametric strategy as

$$
\underline{U}_{h}=\sum_{i=1}^{N_{\text {nodes }}} \lambda_{i}(r, s)\left(\underline{u}_{h}^{(i)}+z \frac{t^{(i)}}{2} \underline{\theta}_{h}^{(i)}\right),
$$

where $\underline{u}_{h}^{(i)}$ represents the displacement at node $i$, and $\underline{\theta}_{h}^{(i)}$ the contribution arising from the rotation of the originally normal vector, hence $\underline{\theta}_{h}^{(i)} \cdot \underline{a}_{3}^{(i)}=0$. Note that this corresponds to a discretization of Reissner-Mindlin kinematics, here only described in a linearized - small displacement - framework as we will use the linear eigenmodes.

The discrete variational formulation is then inferred from the $3 \mathrm{D}$ formulation by using these specific discrete displacements in the 3D integrals, after transforming the elastic tensor to take into account the so-called plane stress assumption, namely,

$$
\Sigma^{z z}=\underline{g}^{z} \cdot \underline{\underline{\Sigma}} \cdot \underline{g}^{z}=0
$$


denoting by $\left(\underline{g}_{r}, \underline{g}_{s}, \underline{g}_{z}\right)$ the covariant basis associated with the local coordinates $(r, s, z)$, i.e. taking in (16)

$$
\underline{g}_{r}=\underline{x}_{, r}, \quad \underline{g}_{s}=\underline{x}_{, s}, \quad \underline{g}_{z}=\underline{x}_{, z},
$$

and with $\left(g^{r}, g^{s}, g^{z}\right)$ the corresponding contravariant basis, see [18] for more details. The internal virtual work is then obtained by integrating matrix-vector products of the type

$$
\stackrel{\sim}{t}^{t} \underset{\sim}{H} \cdot \underset{\sim}{\delta e}
$$

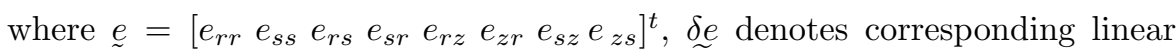
variations, and

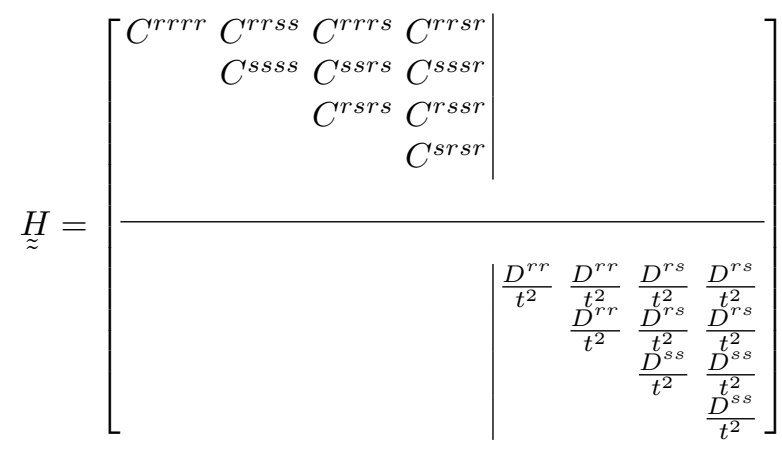

with

$C^{i j k l}=\frac{E}{2(1+\nu)}\left(g^{i k} g^{j l}+g^{i l} g^{j k}+\frac{2 \nu}{1-\nu} g^{i j} g^{k l}\right), \quad D^{i j}=\frac{2 E}{1+\nu} g^{i j}, \quad \forall i, j, k, l=r, s$,

where $E$ and $\nu$ respectively denote Young's modulus and Poisson's ratio, and $g^{i j}=g^{i} \cdot g^{j}$ are the contravariant components of the metric tensor. Note that we do not use symmetrized definitions of the strain vector here - as is classically done in the finite element literature - and the corresponding form of the constitutive matrix. This is because we need to deal with non-symmetric tensors for the computations of the nonlinear stiffness coefficients in (14)-(15), since the tensor $\underline{\underline{\nabla}}^{t} \underline{\Phi}_{i} \cdot \underline{\underline{\nabla}} \underline{\Phi}_{j}$ is non-symmetric when $i \neq j$.

As is well-known, shell finite elements suffer from very serious numerical pathologies when directly discretizing standard kinematical assumptions - namely, when using so-called "displacements-based elements"- see [18] and references therein. In particular, numerical locking is bound to drastically affect the finite element solution of thin shell problems whenever the bending energy is substantial within the total strain energy, unless special measures are taken. In order to circumvent these phenomena without compromising the consistency and stability as regards the membrane and shear energy contributions - hence, allowing to accurately represent the rich diversity of physical behavior that can be encountered in shell structures - we will use specific shell elements called MITC elements ("Mixed Interpolation of Tensorial Components"). These elements are based on the above-described principles, albeit 


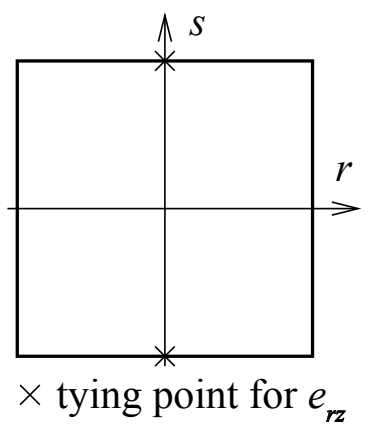

Fig. 1 MITC4 shell element tying points ( $e_{s z}$ tying points obtained by symmetry)

the strain components are not taken as directly computed from the displacements. Instead, each strain component in the $(r, s, z)$ coordinate system is re-interpolated within every element, according to a specific rule based on given points - called the "tying points" - at which the strains are exactly calculated $[18,24]$. In the case of the 4-node quadrilateral MITC4 element, only the transverse shear components $\left(e_{r z}, e_{s z}\right)$ are re-interpolated, based on tying points located at opposing mid-edges, see Figure 1. Therefore, we substitute for the above displacement-based strain vector the modified expression

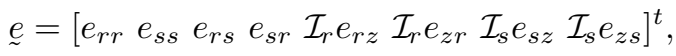

$\mathcal{I}_{r}$ and $\mathcal{I}_{s}$ denoting the interpolation operators, and likewise for the linear variations. In order to compute the coefficients $g_{i j}^{p}$ and $h_{i j k}^{p}$ consistently with the finite element procedure, we thus need to modify the components of the second-order tensors $\underline{\nabla}^{t} \underline{\Phi}_{i} \cdot \underline{\nabla} \underline{\Phi}_{j}$ and $\underline{\nabla} \underline{\Phi}_{p}+\underline{\nabla}^{t} \underline{\Phi}_{p}$ in the exact same manner as $\underline{\underline{e}}$. Note that here the eigenmodes have shape functions as prescribed in (17), namely,

$$
\underline{\Phi}_{j}=\sum_{i=1}^{N_{\text {nodes }}} \lambda_{i}(r, s)\left(\underline{\Phi}_{j}^{(i), \text { depl }}+z \frac{t^{(i)}}{2} \underline{\Phi}_{j}^{(i), r o t}\right),
$$

where $\underline{\Phi}_{j}^{(i), \text { depl }}$ is the displacement part and $\underline{\Phi}_{j}^{(i), \text { rot }}$ the rotation part. Then the counterpart of the strain vector for a product $\underline{\underline{\nabla}}^{t} \underline{\Phi}_{i} \cdot \underline{\underline{\nabla}} \underline{\Phi}_{j}$ reads

$\left[\underline{\Phi}_{i, r} \cdot \underline{\Phi}_{j, r} \underline{\Phi}_{i, s} \cdot \underline{\Phi}_{j, s} \underline{\Phi}_{i, r} \cdot \underline{\Phi}_{j, s} \underline{\Phi}_{i, s} \cdot \underline{\Phi}_{j, r} \mathcal{I}_{r}\left(\underline{\Phi}_{i, r} \cdot \underline{\Phi}_{j, z}\right) \mathcal{I}_{r}\left(\underline{\Phi}_{i, z} \cdot \underline{\Phi}_{j, r}\right) \mathcal{I}_{s}\left(\underline{\Phi}_{i, s} \cdot \underline{\Phi}_{j, z}\right) \mathcal{I}_{s}\left(\underline{\Phi}_{i, z} \cdot \underline{\Phi}_{j, s}\right)\right]^{t}$,

while for $\underline{\nabla} \underline{\Phi}_{p}+\underline{\nabla}^{t} \underline{\Phi}_{p}$ we have

$$
\begin{aligned}
& {\left[\underline{2}_{r} \cdot \underline{\Phi}_{p, r} 2 \underline{g}_{s} \cdot \underline{\Phi}_{p, s}\left(\underline{g}_{r} \cdot \underline{\Phi}_{p, s}+\underline{g}_{s} \cdot \underline{\Phi}_{p, r}\right)\left(\underline{g}_{r} \cdot \underline{\Phi}_{p, s}+\underline{g}_{s} \cdot \underline{\Phi}_{p, r}\right) \ldots\right.} \\
&\left.\ldots \mathcal{I}_{r}\left(\underline{g}_{r} \cdot \underline{\Phi}_{p, z}+\underline{g}_{z} \cdot \underline{\Phi}_{p, r}\right) \mathcal{I}_{r}\left(\underline{g}_{r} \cdot \underline{\Phi}_{p, z}+\underline{g}_{z} \cdot \underline{\Phi}_{p, r}\right) \mathcal{I}_{s}\left(\underline{g}_{s} \cdot \underline{\Phi}_{p, z}+\underline{g}_{z} \cdot \underline{\Phi}_{p, s}\right) \mathcal{I}_{s}\left(\underline{g}_{s} \cdot \underline{\Phi}_{p, z}+\underline{g}_{z} \cdot \underline{\Phi}_{p, s}\right)\right]^{t},
\end{aligned}
$$

where the derivatives of the mode vectors are directly obtained by differentiating in (19). 
The corresponding computational procedures have been implemented in the SHELDDON software developed at Inria. The resulting code has been thoroughly tested by comparing the results obtained for a large set of coefficients with those obtained by the indirect method proposed by Muravyovv and Rizzi [15], which has been applied within the same computational framework. Similar results up to machine precision have been obtained, hence validating the direct numerical computation of non-linear coefficients.

We now turn to an application on a shell structure. A shallow hyperbolic paraboloid panel is selected as an illustrative example. The convergence of nonlinear coefficients accuracy will be discussed. Then, reduced-order models are used for computing the nonlinear response of the structure in the vicinity of its first eigenfrequency, both in free and forced vibrations.

\section{Application : a hyperbolic paraboloid panel}

The general methodology given in the previous section is now applied with a practical test case given by a shallow hyperbolic paraboloid panel. The direct calculation of the nonlinear coupling coefficients is used in order to derive efficient reduced-order models allowing for the quick computation of bifurcation diagrams in free and forced vibrations. Results are illustrated and compared with direct computations using the full FE model, showing the benefit of using the proposed ROM methodology.

\subsection{Panel geometry and convergence study}

A hyperbolic paraboloid panel (hereafter referred to as the HP panel), of lateral dimensions $0.1 \times 0.1 \mathrm{~m}$, thickness $h=1 \mathrm{~mm}$, is selected (see Fig. 2). It is made of an homogeneous isotropic material of Young's modulus $E=2.10^{11} \mathrm{~Pa}$, Poisson ratio $\nu=0.3$ and density $\rho=7800 \mathrm{~kg} / \mathrm{m}^{3}$. The two radii of curvature are such that $R_{x}=-R_{y}=1 \mathrm{~m}$, so that the maximum height of the panel is 1.3 $\mathrm{mm}$, comparable to the thickness $h$. The four edges are clamped by imposing a vanishing displacement for the three displacements $(u, v, w)$ and similarly for the two rotations.

A modal analysis is first performed in order to compute the eigenfrequencies and eigenmodes. Figure 3 shows the eigenvectors $\underline{\Phi}_{p}(x, y)$, for $\mathrm{p}=1,2,6,22$ and 224 (the modes being sorted according to increasing frequencies). The three displacement components $(u, v, w)$ are shown in each row. The panel is shallow so that its bending and membrane behavior is close to that of a plate. One can observe that the low-frequency modes have a behavior that is mostly transverse, the in-plane displacements being one order of magnitude smaller than $w$. On the other hand, above a given frequency, eigenmodes having a behavior that is mostly tangential - where the out-of-plane displacement is one order of magnitude smaller than the in-plane displacement - start appearing. The two 


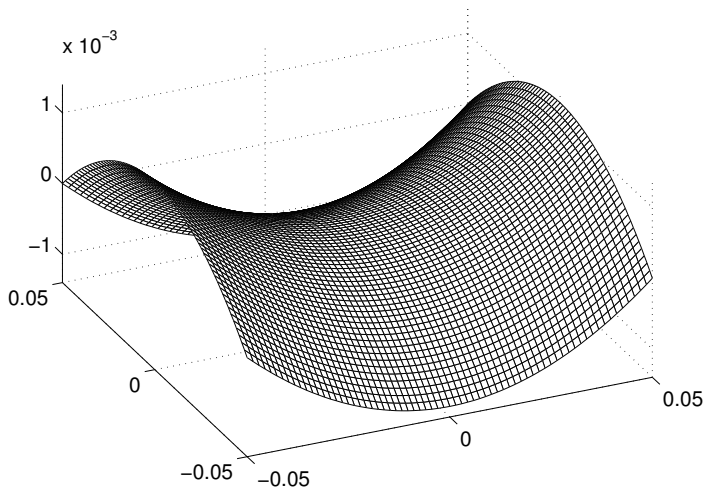

Fig. 2 Hyperbolic paraboloid panel used for the simulations, mesh composed of 4624 quadrangles $\left(N_{n}=4761\right.$ nodes $)$.

families are denoted as B-modes for the modes that are mostly transverse, and M-modes for the mostly tangential. This terminology is chosen in reference to so-called bending- and membrane-dominated asymptotic behaviors, see [18]. In our case the structure considered is statically membrane-dominated due to the clamped boundary conditions. However, the associated eigenproblem does not asymptotically enjoy the compactness properties that characterize a standard structural mechanics eigenproblem. A consequence of this is that - upon decreasing the thickness parameter - the fundamental eigenfrequency does not tend to a finite value, and in general an essential spectrum is found in the asymptotic limit [25]. In addition, the lowest eigenmodes retain a finite proportion of bending energy, while the actually membrane-dominated eigenmodes - energy-wise - correspond to much higher frequencies [26], hence, the B- and M-mode terminology.

In Fig. 3, modes 1,2, 6 and 22 belong to the B-modes family whereas mode 224 belongs to the M-modes. For the B-modes, the mode shape for the transverse displacement $w$ resembles that of a clamped plate. One can see for instance that modes 1,6 and 22 are both symmetric with respect to the $x$ - and $y$-axis. This symmetry property will have a consequence on the nonvanishing value of the nonlinear coefficient. In this regard, we will show in the next section that a strong coupling occurs between all the modes sharing the same symmetry property, and in particular between modes 1, 6 and 22 . Concerning the M-modes, the mode labeled 224 is the first one in increasing frequency order that shows a strong coupling with the fundamental mode and thus will be key in the selection procedure explained in the next section. It appears for a relatively high frequency with $\omega_{224} / \omega_{1}=60$.

The convergence of the eigenfrequencies with the mesh refinement is investigated in Fig. 4. The frequencies of B-modes $p=1,20$ and 40 are considered, as well as the frequency of the M-mode shown in Fig. 3, $p=224$. One can ob- 


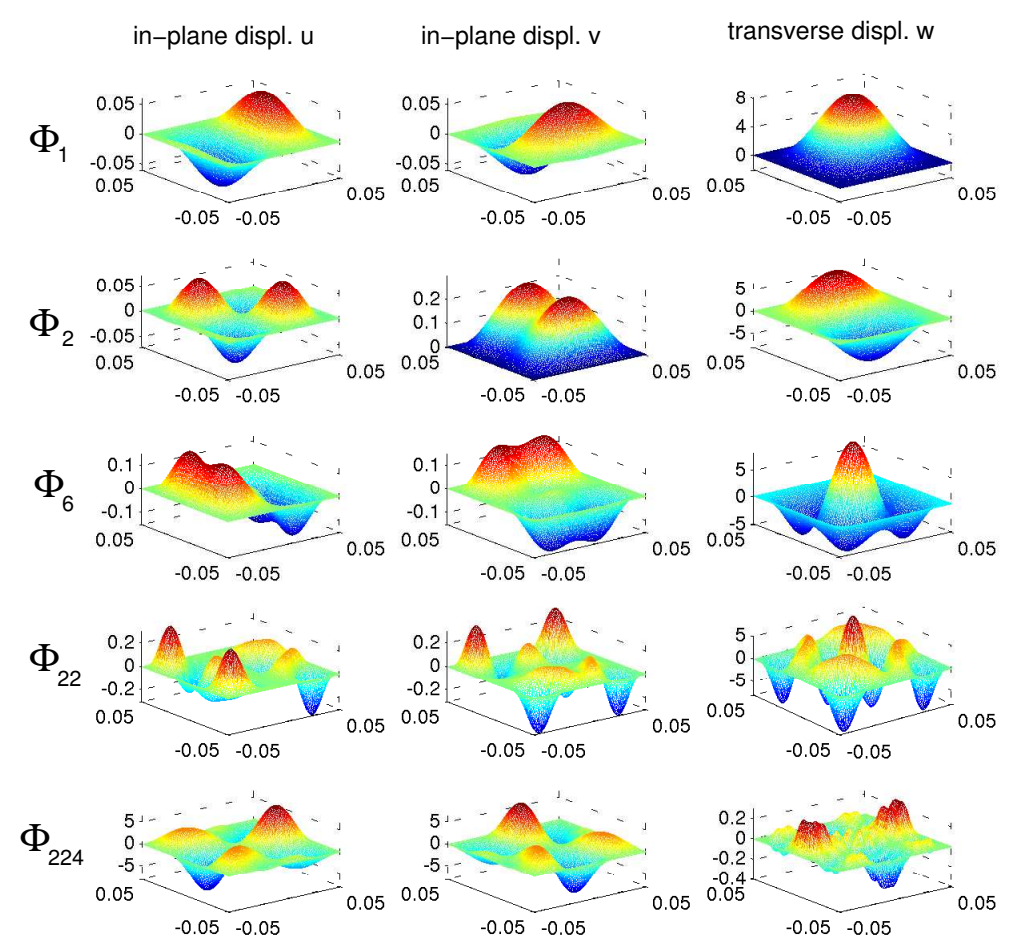

Fig. 3 Five eigenmodes shapes of the HP panel. Each row displays a given mode, with two in-plane displacements $u$ and $v$ and transverse displacement $w$ in successive columns.

serve a rather fast convergence behavior from above, as is classical for finite elements procedures. A good accuracy in the frequencies is obtained starting from $N_{n}=4000$, where $N_{n}$ refers to the number of nodes in the mesh. The convergence for the first M-mode considered, $p=224$, although for a higher frequency, is still very good. This is not surprising, as the convergence velocity is directly related to the wavelength of the mode considered. The convergence of the first M-modes is thus as good as those of the first B-modes as they have comparable wavelengths, although they are of higher frequencies.

The convergence of a selected nonlinear coefficient is reported on in Fig. 5, where the cubic coefficient $h_{p, p, p}^{p}$ appearing in Eq. (15) has been chosen for illustration, and for the same three B-modes $p=1,20$ and 40 . The convergence of an M-Mode (e.g. $p=224$ ) is not shown as the same argument holds for the coupling coefficients as for the eigenfrequencies, namely, the convergence rate is related to wavelength, so that the convergence of $h_{p, p, p}^{p}$ for the first M-modes behaves in the same manner as for the first B-modes. As for the eigenfrequencies, a good convergence is achieved. The numerical values computed here will 


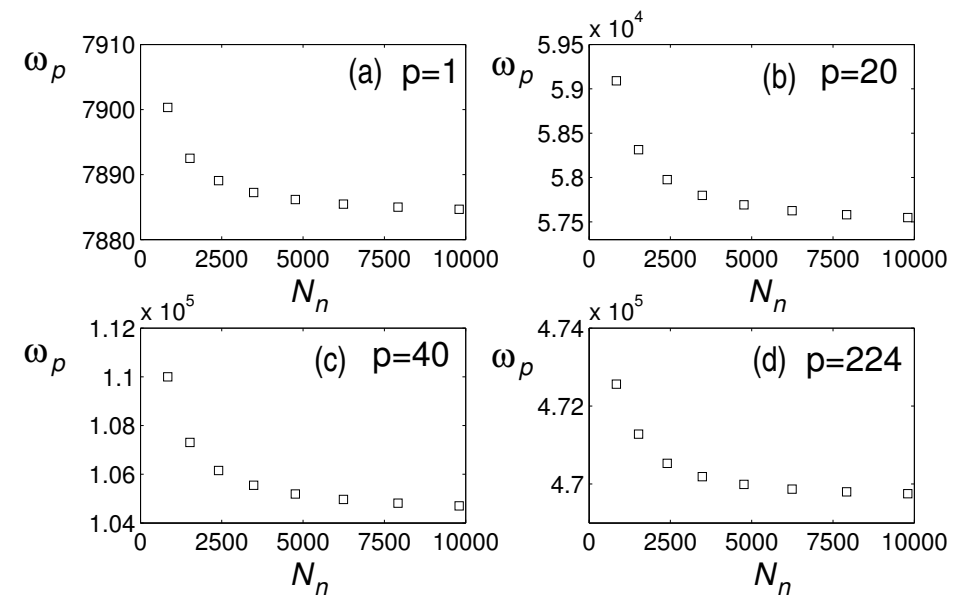

Fig. 4 Convergence of four eigenfrequencies of the HP panel for increasing number of nodes $N_{n}$ in the mesh. (a) $\mathrm{p}=1$, fundamental eigenfrequency, (b) $\mathrm{p}=20$, (c) $\mathrm{p}=40$, (d) $\mathrm{p}=224$, corresponding to the

be used in the next sections for computing dynamical responses of the HP panel, both in free and forced vibrations. As peculiar nonlinear phenomena will be exhibited, a very good accuracy on all the numerical values is needed so as to ensure a good convergence of the reduced-order model. Hence the selected mesh contains a relatively large number of points as compared to the geometric simplicity of the structure. In the remainder of the paper, the mesh of Fig. 2, composed of $N_{n}=4761$ nodes (with 5 degrees-of-freedom (dofs) per nodes), is selected for computing all the quantities needed in the model equations (11).
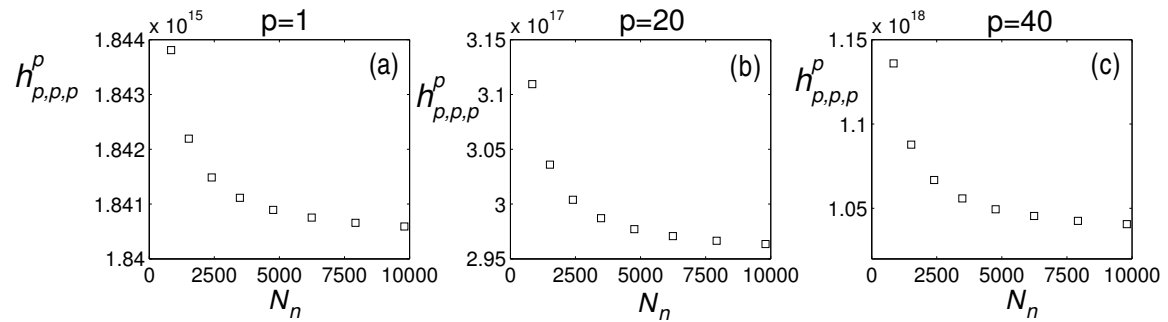

Fig. 5 Convergence of the nonlinear cubic coefficient $h_{p, p, p}^{p}$, with respect to the number of nodes $N_{n}$ of the mesh. (a): p=1, (b) p=20, (c) p=40. 


\subsection{Bifurcation diagram in free vibrations}

The main application of the direct computation of the nonlinear stiffness consists in deriving reduced-order models with a small, selected number of modes, in order to easily compute dynamical characteristics of a shell vibrating at large amplitude. The reduced-order model may be used for either direct time integration, or, as shown next, for computing by a continuation method the bifurcation diagram in the vicinity of a given dynamical state. This method is particularly appealing since it provides a complete picture of the dynamical solutions with stable and unstable states, the latter being unavailable by direct integration.

We start with the bifurcation diagram in free vibration. The structure is left undamped and unforced, and the computation of the family of periodic orbits in the vicinity of the fundamental mode are searched for. As known from nonlinear vibrations, the oscillation period depends on the amplitude of the solution. Following the family of periodic orbits - also known as a nonlinear normal mode (NNM) in the context of Hamiltonian systems [27-32, $5]$ - one can retrieve the oscillation frequency and thus construct the so-called backbone curve of the mode, showing a nonlinear behavior which can be of hardening or softening type.

The construction of the ROM is based on a convergence study with an increasing number of eigenmodes selected in the model. The process is illustrated in Fig. 6. The first physical effect that the reduced-order model must mandatorily capture is the coupling between bending and membrane motions. This means that a sufficient number of M-modes, that have a mostly tangential behavior, has to be retained in the truncation, even though their eigenfrequencies are large. The convergence with this number of high-frequency modes is shown in the insert of Fig. 6, where only the fundamental B-mode is retained with an increasing number of M-modes. The M-modes appear above a given frequency and are numerous, so that a selection criterion must be used. As we are studying the backbone curve of the fundamental mode $(p=1)$, a simple rule consists in selecting the modes that are coupled through a non-vanishing quadratic term of the form $g_{11}^{p}$ to the first mode. As the term $g_{11}^{p}$ creates a monomial of the form $X_{1}^{2}$ into the $p^{\text {th }}$ equation in (11), this means that as soon as energy is present in the first mode, then mode $p$ will be coupled and get a part of this energy via quadratic nonlinear coupling. The membrane modes sharing this property are recorded in Table 1 , where the eigenfrequencies and their ratios to the fundamental one $\left(\omega_{1}=7.886 \cdot 10^{3} \mathrm{rad} . \mathrm{s}^{-1}\right)$ are given.

The backbone curves are computed with a continuation method using a pseudo-arclength scheme implemented in the software AUTO [33]. Before running the continuation, Eqs. (11) are made non-dimensional by dividing the amplitudes by the thickness $h$. The time is also made non-dimensional with timescale $T_{1}=2 \pi / \omega_{1}=7.967 \cdot 10^{-4} \mathrm{~s}$. The output of AUTO is the maximum amplitude of each coordinate $X_{p}$ retained in the model, as well as the period of the orbit. For the insert in Fig. 6, as we are following the first NNM the main contribution is given by $X_{1}$ so that we plot only $\max \left(X_{1}\right)$. This number 


\begin{tabular}{cccc}
\hline $\mathbf{p}$ & $\omega_{p}$ & $\omega_{p} / \omega_{1}$ & $g_{11}^{p}$ \\
\hline $\mathbf{2 2 4}$ & $4.70 \cdot 10^{5}$ & 59.60 & $1.70 \cdot 10^{12}$ \\
$\mathbf{2 8 0}$ & $5.70 \cdot 10^{5}$ & 72.30 & $3.40 \cdot 10^{12}$ \\
$\mathbf{3 4 7}$ & $6.89 \cdot 10^{5}$ & 87.32 & $8.52 \cdot 10^{12}$ \\
$\mathbf{3 7 1}$ & $7.31 \cdot 10^{5}$ & 92.72 & $2.89 \cdot 10^{12}$ \\
$\mathbf{3 8 8}$ & $7.58 \cdot 10^{5}$ & 96.14 & $4.30 \cdot 10^{12}$ \\
$\mathbf{4 2 7}$ & $8.23 \cdot 10^{5}$ & 104.39 & $4.44 \cdot 10^{12}$ \\
$\mathbf{4 9 3}$ & $9.27 \cdot 10^{5}$ & 117.57 & $1.07 \cdot 10^{12}$ \\
$\mathbf{5 0 2}$ & $9.39 \cdot 10^{5}$ & 119.08 & $1.58 \cdot 10^{12}$ \\
$\mathbf{5 5 2}$ & $10.02 \cdot 10^{5}$ & 129.60 & $1.83 \cdot 10^{12}$ \\
\hline
\end{tabular}

Table 1 M-mode family: label $p$ of their appearance in increasing frequency order, radian eigenfrequency $\omega_{p}$ and its ratio to the fundamental $\omega_{1}$, and quadratic coupling coefficients with mode $p=1, g_{11}^{p}$.

is multiplied by the value of the first eigenmode at the center of the panel, $\underline{\Phi}_{1}(0,0)$, so as to directly obtain a physical idea of the maximum vibration amplitude, at the center for the first mode, compared to the thickness $h$. The curves are here followed up to 3 times the thickness.

The first truncation $T_{1}$ shown in the insert of Fig. 6 contains only the fundamental mode, and displays a hardening behavior. The second truncation $\mathrm{T}_{2}$ is obtained by adding the first three M-modes (i.e. $\mathrm{p}=224,280$ and 347) to the truncation. One observes that the hardening behavior is less pronounced, which reveals the effect of adding those modes in order to correctly reproduce the nonlinear vibrating behavior of the panel. The last two truncations, $\mathrm{T}_{3}$ and $\mathrm{T}_{4}$, are almost superimposed and show that the convergence is achieved up to three times the thickness. They are obtained respectively for the first seven $\left(\mathrm{T}_{3}\right)$ and first nine $\left(\mathrm{T}_{4}\right)$ M-modes - given in Table 1 - included in the truncation. In what follows, the seven first modes of Table 1 are selected in order to correctly reproduce the bending-membrane coupling in the reducedorder model.

\begin{tabular}{ccc|ccc}
\hline $\mathbf{p}$ & $\omega_{p}$ & $\omega_{p} / \omega_{1}$ & $\mathbf{p}$ & $\omega_{p}$ & $\omega_{p} / \omega_{1}$ \\
\hline $\mathbf{1}$ & $0.788 \cdot 10^{4}$ & 1.00 & $\mathbf{1 2}$ & $3.785 \cdot 10^{4}$ & 4.80 \\
$\mathbf{2}$ & $1.230 \cdot 10^{4}$ & 1.56 & $\mathbf{1 3}$ & $3.800 \cdot 10^{4}$ & 4.81 \\
$\mathbf{3}$ & $1.230 \cdot 10^{4}$ & 1.56 & $\mathbf{1 4}$ & $4.612 \cdot 10^{4}$ & 5.84 \\
$\mathbf{4}$ & $1.719 \cdot 10^{4}$ & 2.18 & $\mathbf{1 5}$ & $4.612 \cdot 10^{4}$ & 5.84 \\
$\mathbf{5}$ & $2.102 \cdot 10^{4}$ & 2.67 & $\mathbf{1 6}$ & $4.840 \cdot 10^{4}$ & 6.14 \\
$\mathbf{6}$ & $2.110 \cdot 10^{4}$ & 2.67 & $\mathbf{1 7}$ & $4.843 \cdot 10^{4}$ & 6.14 \\
$\mathbf{7}$ & $2.590 \cdot 10^{4}$ & 3.28 & $\mathbf{1 8}$ & $5.312 \cdot 10^{4}$ & 6.74 \\
$\mathbf{8}$ & $2.590 \cdot 10^{4}$ & 3.28 & $\mathbf{1 9}$ & $5.312 \cdot 10^{4}$ & 6.74 \\
$\mathbf{9}$ & $3.311 \cdot 10^{4}$ & 4.20 & $\mathbf{2 0}$ & $5.770 \cdot 10^{4}$ & 7.31 \\
$\mathbf{1 0}$ & $3.311 \cdot 10^{4}$ & 4.20 & $\mathbf{2 1}$ & $6.111 \cdot 10^{4}$ & 7.75 \\
$\mathbf{1 1}$ & $3.430 \cdot 10^{4}$ & 4.35 & $\mathbf{2 2}$ & $6.131 \cdot 10^{4}$ & 7.77 \\
\hline
\end{tabular}

Table 2 B-modes, radian eigenfrequency $\omega_{p}$ and ratio to the fundamental $\omega_{1}$. 


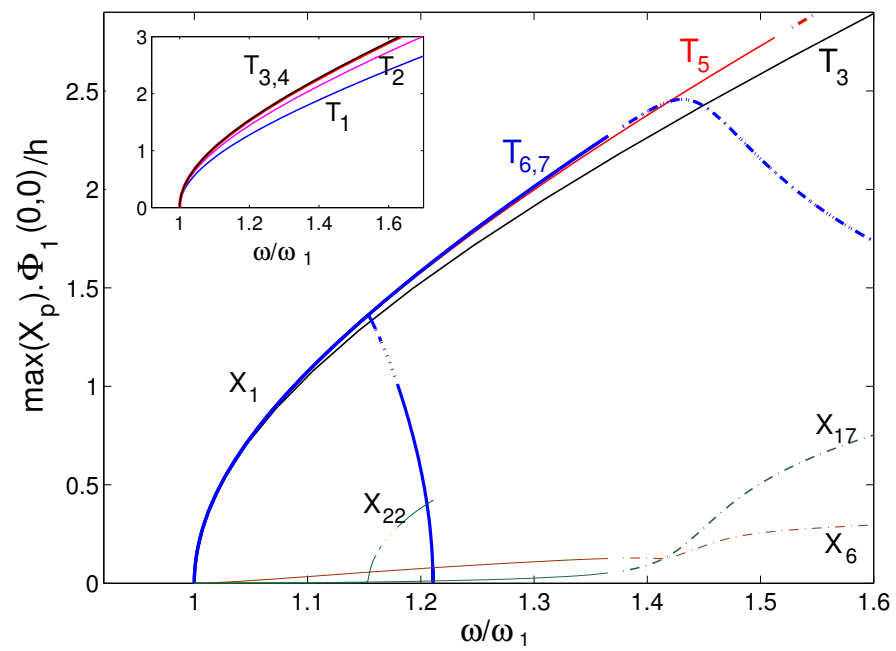

Fig. 6 Backbone curve of the fundamental mode of the HP panel, convergence study. Insert: convergence of the backbone for an increasing number of M-modes and a single B-mode. $\mathrm{T}_{1}$ : fundamental T-mode only $-\mathrm{T}_{2}$ : fundamental T-mode +3 M-modes $(224,280$ and 347$)-$ $\mathrm{T}_{3}$ : fundamental $+7 \mathrm{M}$-modes (modes $371,388,427$ and 493 added) $-\mathrm{T}_{4}$ : fundamental +9 M-modes (502, 522 added). Main figure: Convergence of the backbone curve for an increasing number of B-modes, for a fixed number (7) of M-modes. $\mathrm{T}_{5}$ : B-modes 2 to 6 added $-\mathrm{T}_{6}$ : B-modes 1 to 13 plus 17 and $22-\mathrm{T}_{7}$ : B-modes 1 to $22 . X_{1}$ : main coordinate, $X_{6}$ and $X_{17}$ : two most important non-resonant couplings, $X_{22}$ : internally resonant coordinate. Unstable states represented with dash-dotted lines.

The convergence with the number of transverse modes is studied in the main part of Fig. 6, where the truncation $\mathrm{T}_{3}$ is considered as a starting solution. The transverse modes, with the lowest eigenfrequencies, are given for $p=1$ to 22 in Table 2 . Truncation $\mathrm{T}_{5}$ contains the first six modes of this set (with the seven M-modes already identified and now considered as fixed). We observe that adding the first modes has two effects: first the hardening behavior is influenced and less pronounced for $\mathrm{T}_{5}$ as compared to $\mathrm{T}_{3}$. The second effect is a loss of stability for a limit amplitude value of vibration, computed here for $\mathrm{T}_{5}$ as $2.8 \mathrm{~h}$. This absence of stable periodic orbit is consistent with previous studies on the transition to turbulence for thin plates and shells, see e.g. [34-36], where it has been found that from vibration amplitudes of 2 to $4 h$ (depending on the structure considered), no stable periodic solutions exist anymore, so that the dynamical solution is at least quasi-periodic. The amplitude limit in the case presented here of the HP panel is found to converge to a value of $2.3 h$, as shown by the last two truncations which are superimposed, $\mathrm{T}_{6}$ and $\mathrm{T}_{7}$. Truncation $\mathrm{T}_{7}$ is built by considering all the 22 first modes (with the seven M-modes) shown in Table 2. Among those 22 B-modes, and inspecting the cubic coupling terms $\left\{h_{i j k}^{p}\right\}$, one observes that most of these coefficients vanish, so that a clever truncation can also be produced by con- 
sidering only modes $p$ coupled with the fundamental mode under study, i.e. for which $h_{111}^{p} \neq 0$. Interestingly, the modes featuring this property are those sharing the same symmetry property (in terms of mode shape) as the fundamental, i.e. they are both symmetric with respect to the $x$ - and $y$-axis. The modes of this family in Table 2 are for $p=1,6,11,17$ and 22. Truncation $\mathrm{T}_{6}$ is converged by considering only 13 transverse modes (instead of 22 for $\mathrm{T}_{7}$ ), namely, $p=1$ to 11 , plus 17 and 22 .

In Fig. 6, most of the energy is contained within the first modal coordinate $X_{1}$ as we continue the periodic orbits of the fundamental mode. The three other most important coordinates are also shown in the figure. They correspond to modes sharing the same symmetry property as the fundamental, namely $p=6,17$ and 22 (mode $p=11$ also presents a non-vanishing value, but negligible so that it is not shown in the figure). One can observe that modes 6 and 17 feature a non-resonant coupling with the fundamental $p=1$, as they slowly increase when following the backbone curve. A small amount of energy is transferred through the $h_{111}^{p}$ coupling term, but no commensurability relationship exists between the nonlinear frequencies. On the other hand, a resonant coupling is observed between modes 1 and 22, giving rise to the tongue of internally resonant periodic orbits around $\omega / \omega_{1} \simeq 1.8$, where $X_{1}$ displays a rapid decrease in amplitude, the energy being transferred to $X_{22}$ which increases importantly in the frequency range. These tongues of internal resonance have already been observed in smaller systems [32,37-39] as well as in nonlinear vibrations of plates [36]. Interestingly, it has been emphasized in [36] that when the internal resonance occurs in a narrow interval as here observed, its influence on the frequency response in the forced-damped system is negligible. This will be again ascertained here for the HP panel in the next section.

As a conclusion on the convergence study of the ROM and the backbone curve of the HP panel, we have found that the first NNM (as a family of periodic orbits for the conservative system) is converged for a ROM including 20 linear modes, decomposed into $13 \mathrm{~B}$-modes (mostly transverse), and $7 \mathrm{M}$ modes (mostly tangential). Interestingly, no stable periodic orbits have been found beyond a vibration amplitude of $2.3 h$, in line with previous studies. An internal resonance tongue has been found with mode 22 , albeit it occurs in a very narrow interval, so that its effect on the global dynamics of the forceddamped system is likely to be negligible. This will be confirmed in the next subsection.

\subsection{Bifurcation diagrams for forced and damped vibrations}

The more physical case of the forced vibrations of the damped HP panel is now studied. For the reduced-order model, a diagonal (modal) damping is considered by adding a term of the form $\xi \dot{X}_{p}$ in each oscillator equation (11). This classical damping term is incorporated in the non-dimensional equations, 
where the timescale is selected as $T_{1}$ and the amplitudes are divided by $h$ as in the previous section for the AUTO simulations. Returning to dimensional variables, one understands that the factor $\xi$ corresponds to a damping ratio (dimensionless). It is here set to $\xi=0.05$ for the computations. The forcing is assumed to be pointwise and located at the center of the panel. Its time dependence is harmonic with a forcing radian frequency denoted by $\Omega$. The dimensional force amplitude is denoted by $F$. The ROM is selected from the convergence study on the backbone curve and contains the 20 linear modes identified in the previous section.

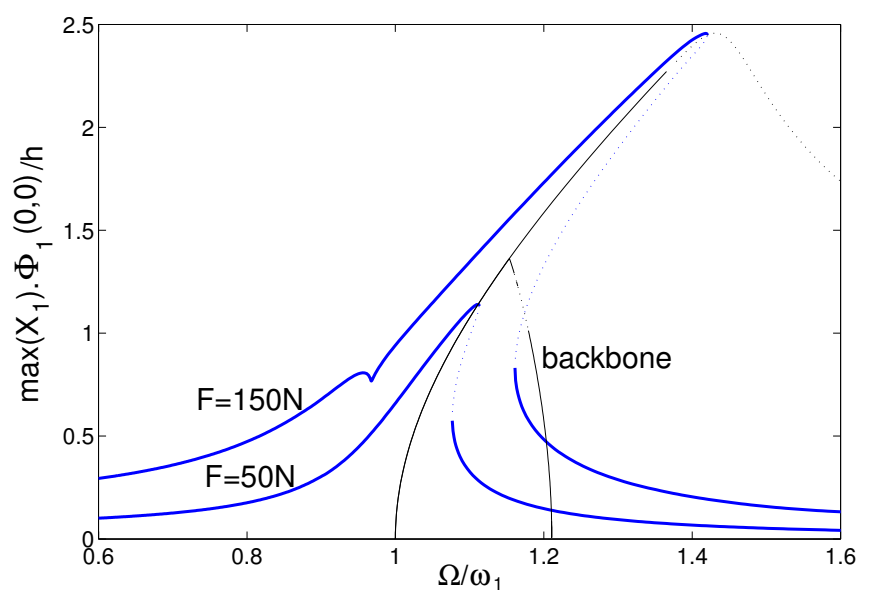

Fig. 7 Frequency-response function of the HP panel, modal damping ratio $\xi=0.05$ for each mode, harmonic pointwise forcing of varying frequency $\Omega$ and force $F$ applied at center of the panel. $F=50 \mathrm{~N}$ and $F=150 \mathrm{~N}$.

Figure 7 shows the results for $F=50 \mathrm{~N}$ and $150 \mathrm{~N}$, together with the backbone curve. The frequency-response functions are obtained by continuation on the ROM, hence showing the stable and unstable states of the system. As the ROM contains a small number of oscillator equations, the simulation time for computing a complete frequency-response is very small, of the order of 5 minutes on a standard computer for the largest models used. Note that this computing time varies with the number of modes considered, but also - and in fact even more - with the complexity of the bifurcation diagram. Nevertheless, this is negligible compared with the computing time required by direct dynamical integration of a finite element solution, see below comparison. Only the maximum of the main coordinate $X_{1}$ is represented in the figure, and the factor $\Phi_{1}(0,0) / h$ is still used to allow a rapid comparison with the maximum vibration amplitude at center with respect to the thickness. For the smallest forcing amplitude, $F=50 \mathrm{~N}$, the frequency-response curve reaches a maximum amplitude around $h$, and a small region of hysteresis is found between the two 
saddle-node bifurcation points. The nonlinear behavior is more pronounced for $F=150 \mathrm{~N}$, with a maximum amplitude of $2.45 \mathrm{~h}$. As expected, the frequencyresponse functions are organized along the backbone curve. However, one can note that for $F=150 \mathrm{~N}$ the upper branch is still stable for vibration amplitudes larger than $2.3 h$, whereas the backbone curve did not present stable periodic orbits above that amplitude. This difference is brought by the presence of damping which slightly modifies the stability properties of the manifolds at large amplitude. As already shown in [36], one has to consider very small values of the damping ratio (of the order of 0.001 ) to recover the stability prediction given by the conservative case. When damping is added, slight variations are expected, as observed here. One can also remark that the branch of internal resonance found in the conservative case is completely undetected in the forced-damped case. Finally, for $F=150 \mathrm{~N}$, a small peak is observed around $\Omega / \omega_{1} \simeq 1$. This will be explained later when commenting Fig. 9 .

In the case of forced and damped vibrations, the amplitude responses predicted by the ROM and shown in Fig. 7 can be compared to direct simulations on the full FE model, so as to ascertain the quality of the ROM and its ability to predict the correct amplitude values. To that purpose, the same pointwise harmonic forcing is considered for the full FE model which is integrated in time with a standard Newmark scheme. The same damping law is selected by adding a damping matrix of the form $\mathbf{C}=\alpha \mathbf{M}$, where $\mathbf{M}$ is the mass matrix and $\alpha$ a damping parameter which is prescribed so that the damping coefficient in the non-dimensional modal dynamics equations is $\xi=0.05$. In order to be able to obtain stable states on the upper branch of the frequency-response, for each excitation frequency the initial condition is selected as the final state of the numerical integration associated with the previous forcing frequency (and for the lowest frequency the initial condition is the structure at rest). As the basin of attraction shrinks when one travels along the upper branch of the response, this strategy allows finding solutions up to the largest vibration amplitude. Finally, for each $\Omega$, the permanent regime is awaited and the maximum amplitude of the response at center is recorded.

The comparison is shown in Fig. 8 for $F=50 \mathrm{~N}$. The time integration for the full model is computed, for each $\Omega$, on a total time of 50 excitation periods, and the time step is selected so as to have 40 points per excitation period. This results in a simulation time of about 2 hours (on the same standard computer) for obtaining each point on the curve. Note that Fig. 8(a) does not compare exactly the same data. For the full FE model, the maximum amplitude of the transverse displacement at the center is shown, whereas for the ROM the continuation software AUTO gives the maximum amplitude of each coordinate $X_{p}$, without their relative phases, so that one is not able to reconstruct precisely the complete transverse displacement by adding all modal coordinates according to Eq. (10). For that reason only the main coordinate $X_{1}$ is considered in Fig. 8(a). In order to precisely compare the same quantities, Figs. 8(b) and (c) show the phase portraits obtained for the full model versus the ROM, obtained by plotting $\dot{w}(0,0, t)$ versus $w(0,0, t)$. For the ROM, a direct integration has been implemented on the oscillator equations, with a 

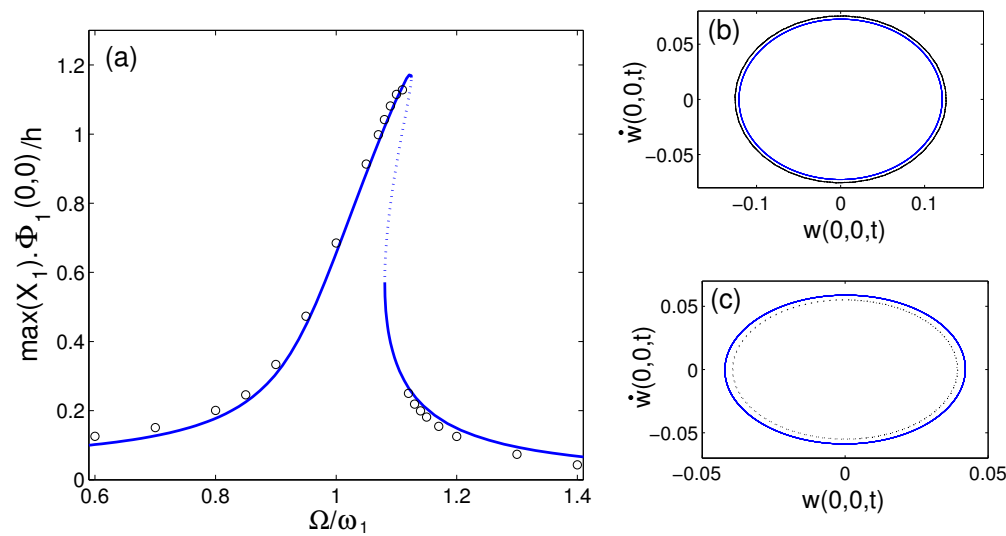

Fig. 8 Forced response of the HP panel, forcing amplitude $F=50 \mathrm{~N}$, comparison between ROM and full FE model. (a): frequency-response function. Solid and dotted lines: stable and unstable solutions, maximum of first coordinate $X_{1}$ of the ROM. Circles (o): maximum amplitude value of the displacement at center $w(0,0, t)$ inferred from a direct time simulation of the full FE model. (b): phase portrait $\dot{w}(0,0, t)$ versus $w(0,0, t)$ for the full FE model (black dotted line) and the ROM (blue solid line), for $\Omega=0.6 \omega_{1}$. (c): idem for $\Omega=1.4 \omega_{1}$.

time-discretization following a Störmer-Verlet (or leap-frog) scheme [40]. Two excitation frequencies have been selected: $\Omega=0.6 \omega_{1}$ for Fig. $8(\mathrm{~b})$, and $\Omega=1.4 \omega_{1}$ for Fig. 8(c).

The excellent agreement between the two models evidenced in Fig. 8 underlines that most of the vibratory energy is contained within the first coordinate $X_{1}$, whereas the others are negligible. However, one should beware of not discarding the other coordinates in a ROM, as shown for example in the convergence study where increasing the number of modes has led to a significant change in the type of nonlinearity. As already discussed in other papers, see e.g. [41-44,9], the change can be even more significant and neglecting too abruptly higher modes could lead to predicting a hardening-type nonlinearity whereas the real behavior is of the softening type. Returning to Fig. 8(a), one can observe that the ROM seems to slightly underpredict the maximal amplitude before the resonance, and overpredict after the resonance. Reconstructing the complete displacement in Fig 8(b-c) shows that this effect, although reduced, is still slightly present. However, one can conclude from this simulation that for this level of vibration amplitude the two models are in excellent agreement, which means that the ROM has correctly captured the physics of the shell vibrations.

The comparison for a larger forcing amplitude, $F=150 \mathrm{~N}$, is shown in Fig. 9(a) where a global quantitative agreement is found. Once again the ROM seems to slightly underestimate the vibration amplitude before the resonance, albeit when reconstructing the complete displacement at the center for $\Omega=0.85 \omega_{1}$, one can see in Fig. 9 (b) that the two periodic orbits are almost superimposed. After the resonance, for larger excitation frequencies the ROM 

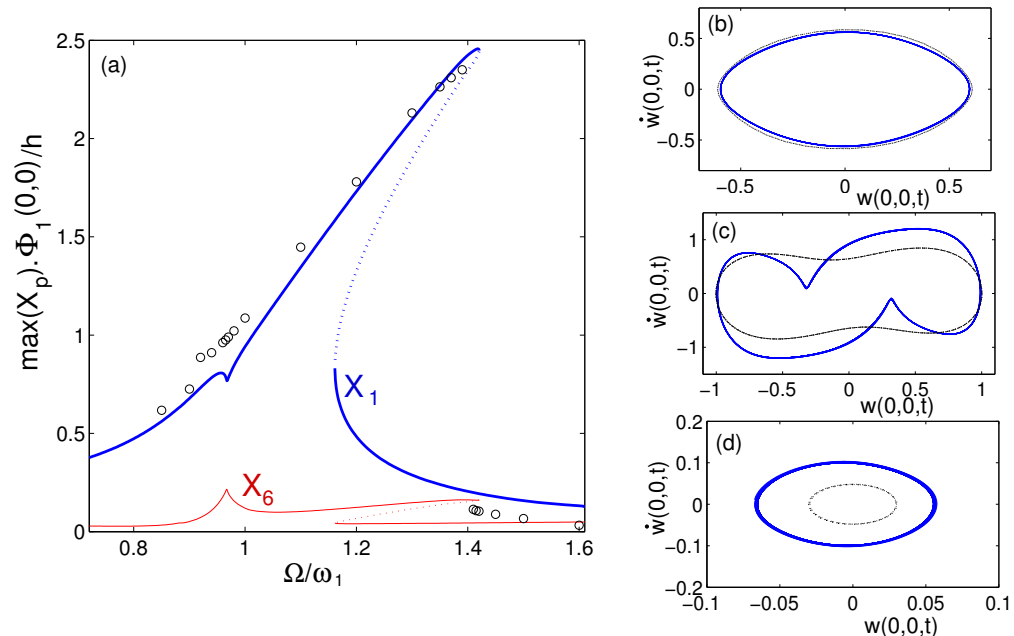

Fig. 9 Forced response of the HP panel, forcing amplitude $F=150 \mathrm{~N}$, comparison between ROM and full FE model. (a): frequency-response function. Solid and dotted blue lines: stable and unstable solutions, maximum of first coordinate $X_{1}$ of the ROM. Red lines: maximum of sixth coordinate $X_{6}$. Circles (o): maximum amplitude value of the displacement at center $w(0,0, t)$ inferred from a direct time simulation of the full $\mathrm{FE}$ model. (b): phase portrait $\dot{w}(0,0, t)$ versus $w(0,0, t)$ for the full FE model (black dotted line) and the ROM (blue solid line), for $\Omega=0.85 \omega_{1}$, (c): $\Omega=0.97 \omega_{1},(\mathrm{~d}): \Omega=1.6 \omega_{1}$.

overestimates the vibration amplitude, although one can note by inspecting Fig. 9(d) for $\Omega=1.6 \omega_{1}$ that the discrepancy is less pronounced when adding all contributions of the involved modes. Finally, the most striking point of this frequency-response curve is the appearance of a secondary peak around $\Omega \simeq \omega_{1}$. The physical interpretation of this is given by the ROM, which shows that an internal resonance occurs with the mode $p=6$, as the sixth coordinate $X_{6}$ gains a non-negligible amplitude response, see Fig. 9(a). This resonance is due to the fact that the nonlinear oscillation frequency of the first mode is exactly equal to one third of the sixth one, hence it is called a 3:1 resonance. Interestingly, the complete FE model also displays a change in behavior around this frequency, which shows that the phenomenon is similarly captured by the complete model. The apparent discrepancy in Fig. 9(a) on the amplitude predicted by the two models is due to the fact that only $X_{1}$ is represented for the ROM. Adding all coordinates in a direct computation of the 20-modes ROM and comparing the phase portrait $(w(0,0, t), \dot{w}(0,0, t))$, in Fig. 9(c) for $\Omega=0.97 \omega_{1}$ shows that the maximum amplitude in displacement is coincident for the two models. However, a difference subsists between the two models, as shown in the detailed phase portraits, and one can observe that the 3:1 resonance is more pronounced for the ROM than for the full model. This might be ascribed to the fact that the 3:1 resonance is activated when the nonlinear frequencies share the perfect 3:1 relationship, and the complete 
model may feature a slight difference in the evolution of the higher frequencies versus vibration amplitude.

Summarizing the results obtained in this section, we can conclude that the ROM is reliable and provides fast access to numerous results that are beyond reach for a full FE model. In particular, stable as well as unstable states are computable at a far reduced simulation cost. Having access to all modal coordinates is also meaningful for physical interpretations of internal resonances as the energy exchanges are then easily observable. Hence, the method shows its ability in performing efficient model prediction for nonlinear, resonant vibrations of thin shells. The last section is devoted to the maximal reduction one is able to achieve in the framework of nonlinear frequencyresponse, by using another change of coordinates and a single, asymptotic, nonlinear normal mode (NNM).

\subsection{Maximal reduction - Single NNM}

As discussed in the previous section, a ROM composed of 20 linear modes has been found to be able to accurately reproduce the free vibration diagram as well as the forced-damped frequency response functions of the HP panel up to vibration amplitudes of 2.5 times the thickness, in the vicinity of the fundamental eigenfrequency. As already emphasized, most of the energy is then contained within the first coordinate $X_{1}$, but a too abrupt truncation considering only the first linear mode is known to potentially lead to erroneous predictions, as discussed for example in $[45,46,41-43,47,44,48,9]$. This truncation problem, which may in the worst cases lead to predicting a hardening-type nonlinearity whereas the real behavior is of the softening type, is known to be related to the problem of the loss of invariance of the linear manifold. Nonlinear normal modes (NNMs), defined as invariant manifolds that are tangent to the linear eigenspaces at the origin [49], allow to remedy this problem and thus predict the correct type of nonlinearity for the same complexity at hand $[44,50]$.

Asymptotic NNMs computed via normal form theory provide an operative, efficient framework for further reducing ROMs built on linear normal modes to a single NNM and thus a single nonlinear oscillator equation. The method is fully described in $[44,50]$ and here briefly recalled. Based on the modal equations (11) with the coupling coefficients computed with our above approach, a nonlinear change of coordinates can first be defined to transform the modal coordinates $X_{p}$ - together with the velocity $Y_{p}=\dot{X}_{p}$ - to new, normal coordinates, $\left(R_{p}, S_{p}\right)$ that describe the dynamics in the curved, invariant-based span of the phase space. Following an asymptotic expansion, the nonlinear change of coordinate is defined in the generic form

$$
\begin{gathered}
X_{p}=R_{p}+\mathcal{P}_{p}\left(R_{i}, S_{j}\right), \\
Y_{p}=S_{p}+\mathcal{Q}_{p}\left(R_{i}, S_{j}\right),
\end{gathered}
$$


where $\mathcal{P}_{p}$ and $\mathcal{Q}_{p}$ are third-order polynomials, the analytical expressions of which are given in [44] for the undamped case and in [50] for the damped case. Once the nonlinear change of coordinates has been computed, one just has to select the excited NNM, here the fundamental one $\left(R_{1}, S_{1}\right)$, and let all other normal coordinates vanish, so as to obtain a single nonlinear oscillator equation for $\left(R_{1}, S_{1}\right)$ that properly takes into account all the non-resonant couplings.

The performance of three different ROMS are compared in Fig. 10 for their ability to predict the nonlinear frequency-response curve of the HP panel. The first ROM is that selected in the previous sections, composed of 20 linear modes and taken as reference. Two other ROMs composed of a single oscillator equation are compared. The first one is derived by retaining only the fundamental linear mode $X_{1}$ in the truncation, whereas the second one is composed of a single NNM oscillator-equation, that has been built from the reference model with 20 linear modes. The two different excitation amplitudes of $50 \mathrm{~N}$ and 150 $\mathrm{N}$ are still selected so as to draw comparisons in a moderately nonlinear case (vibration amplitudes of the order of the thickness $h$ ) as well as in a more strongly nonlinear case (up to $2.5 h$ ).
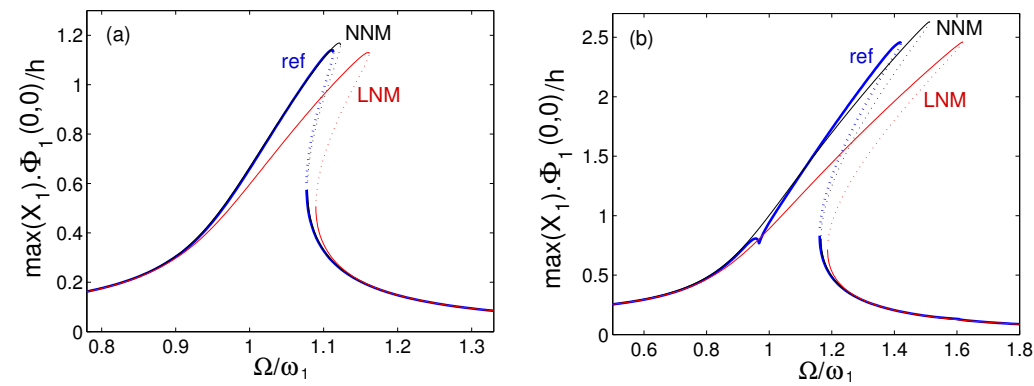

Fig. 10 Frequency-response functions for the forced response of the HP panel, (a): forcing amplitude $F=50 \mathrm{~N}$ and (b): $150 \mathrm{~N}$. Comparison between ROMS: blue (ref): 20 linear modes, red (LNM): a single linear mode and black (NNM): a single nonlinear normal mode.

Fig. 10(a) shows that the single, linear, mode truncation gives an erroneous prediction of the type of non-linearity so that the frequency-response curve, which follows the backbone curve, tends to depart from the reference solutions as soon as the nonlinearities comes into play. This mis-prediction is due to the fact that all the important modes identified in the convergence study are here discarded. On the other hand, the ROM composed of a single NNM has all these slave modes embedded in the nonlinear change of coordinates, and thus can predict the correct behavior, showing an excellent agreement with the reference solution.

Increasing the forcing amplitude to $150 \mathrm{~N}$, Fig. 10(b) shows that the NNMbased ROM, although giving a globally accurate prediction as compared to the 
single linear mode truncation, slightly overestimates the maximal amplitude. Secondly it completely misses the 3:1 internal resonance with the second mode. This is a known drawback of this method that assumes no internal resonance relationships. However, the discrepancy in amplitude remains small and the global prediction, for vibration amplitudes up to $2.5 h$, is very good, for a single oscillator equation, which means that the continuation curves are computed almost immediately.

\section{Conclusion}

A direct method has been presented for computing the nonlinear stiffness in geometric nonlinear vibration of thin shells discretized by finite elements. Analytical expressions of the nonlinear coupling coefficients have been expressed from the internal elastic energy by using a modal expansion for the displacement. These formulas have been implemented in a FE code within the framework of MITC shell elements. The method gives fast and reliable computations for these coefficients, the accuracy of which has been validated by comparison with an indirect method. In this paper we have specifically used this approach with the 4-node MITC4 quadrilateral element, but it can also be applied to other elements of the MITC family in a very straightforward manner.

The computation of these coefficients can be used for deriving reducedorder models of great accuracy, allowing for precise prediction of nonlinear vibration characteristics of shell models. In this paper, the ROMs constructed from the nonlinear coefficients are used with a numerical continuation method in order to produce the complete bifurcation diagrams depicting the nonlinear vibrations of the shell, both in free and forced vibrations. This methodology is particularly appealing as it provides access to stable as well as unstable states of the system, which is of great interest in a predictive perspective.

A converged ROM including 20 linear modes has been shown to predict with excellent accuracy the resonant response of a HP panel in the vicinity of its fundamental frequency. The vibratory response has been compared to direct simulations of the full FE model, showing very good agreement for computational times that are incomparable. Finally, it has been shown that an ultimate reduction process can be derived thanks to the asymptotic NNMs formalism, allowing reduction to a single oscillator equation. Using a single NNM, one is able to compute the correct response of the shell, although one must be aware that internal resonances are not taken into account, so that some subtle behavior in the frequency-response curve may be missed.

The methodology presented in this paper is fast and reliable and the ROMs may be used in a variety of contexts. It also paves the way for further fine numerical studies of bifurcation diagrams for shells of arbitrary complex geometry. Such studies will also allow a more extensive assessment of the criterion used in the present paper to select the important modes in the ROM, which can lead to an automatized selection procedure. 


\section{References}

1. A. H. Nayfeh. Nonlinear interactions: analytical, computational and experimental methods. Wiley series in nonlinear science, New-York, 2000.

2. M. Amabili. Nonlinear vibrations and stability of shells and plates. Cambridge University Press, 2008.

3. R. Seydel. Practical bifurcation and stability analysis. Springer, New-York, 2010. Third edition.

4. B. Krauskopf, H. Osinga, and J. Galán-Vioque. Numerical continuation methods for dynamical systems. Springer, 2007.

5. A. H. Nayfeh and D. T. Mook. Nonlinear oscillations. John Wiley \& sons, New-York 1979.

6. R. Lewandowski. Computational formulation for periodic vibration of geometrically nonlinear structures, part II: numerical strategy and examples. International Journal of Solids and Structures, 34:1949-1964, 1997.

7. H. N. Arafat and A. H. Nayfeh. Non-linear responses of suspended cables to primary resonance excitation. Journal of Sound and Vibration, 266:325-354, 2003.

8. M. Amabili, F. Pellicano, and M. P. Païdoussis. Non-linear dynamics and stability of circular cylindrical shells containing flowing fluid, part II: large-amplitude vibrations without flow. Journal of Sound and Vibration, 228(5):1103-1124, 1999.

9. C. Touzé, M. Amabili, and O. Thomas. Reduced-order models for large-amplitude vibrations of shells including in-plane inertia. Computer Methods in Applied Mechanics and Engineering, 197(21-24):2030-2045, 2008.

10. M. Amabili. A comparison of shell theories for large-amplitude vibrations of circular cylindrical shells: Lagrangian approach. Journal of Sound and Vibration, 264:1091$1125,2003$.

11. L. Kurpa, G. Pilgun, and M. Amabili. Nonlinear vibrations of shallow shells with complex boundary: R-functions method and experiments. Journal of Sound and Vibration, 306(3-5):580-600, 2007.

12. A. Lazarus, O. Thomas, and J.-F. Deü. Finite element reduced order models for nonlinear vibrations of piezoelectric layered beams with applications to NEMS. Finite Elements in Analysis and Design, 49:35-51, 2012.

13. F. Boumediene, A. Miloudi, J.M. Cadou, L. Duigou, and E.H. Boutyour. Nonlinear forced vibration of damped plates by an asymptotic numerical method. Computers and Structures, 87(2324):1508-1515, 2009.

14. F. Boumediene, L. Duigou, E.H. Boutyour, A. Miloudi, and J.M. Cadou. Nonlinear forced vibration of damped plates coupling asymptotic numerical method and reduction models. Computational Mechanics, 47(4):359-377, 2011.

15. A.A. Muravyov and S.A. Rizzi. Determination of nonlinear stiffness with application to random vibration of geometrically nonlinear structures. Computers and Structures, 81:1513-1523, 2003

16. M. Mignolet and C. Soize. Stochastic reduced-order models for uncertain geometrically nonlinear dynamical systems. Computer Methods in AppL. Mech. Engrg., 197:39513963, 2008.

17. M. Mignolet, A. Przekop, S.A. Rizzi, and S.M. Spottswood. A review of indirect/nonintrusive reduced-order modeling of nonlinear geometric structures. Journal of Sound and Vibration, 332:2437-2460, 2013.

18. D. Chapelle and K.J. Bathe. The Finite Element Analysis of Shells - Fundamentals. Springer, second edition, 2011.

19. L. Meirovitch. Computational Methods in Structural Dynamics. Sijthoff and Noordhoff, The Netherlands, 1980

20. G. Berkooz, P. Holmes, and J.L. Lumley. The proper orthogonal decomposition in the analysis of turbulent flows. Annual review of Fluid Mechanics, 25:539-575, 1993.

21. P. Krysl, S. Lall, and J.E. Marsden. Dimensional model reduction in non-linear finite element dynamics of solids and structures. International Journal for numerical methods in engineering, 51:479-504, 2001.

22. M. Amabili and C. Touzé. Reduced-order models for non-linear vibrations of fluid-filled circular cylindrical shells: comparison of pod and asymptotic non-linear normal modes methods. Journal of Fluids and Structures, 23(6):885-903, 2007. 
23. K.J. Bathe. Finite Element Procedures. Prentice-Hall, New-Jersey, 1996.

24. E.N. Dvorkin and K.J. Bathe. A continuum mechanics based four-node shell element for general non-linear analysis. Eng. Comput., 1:77-88, 1984.

25. E. Sanchez-Palencia. Asymptotic and spectral properties of a class of singular-stiff problems. J. Math. Pures Appl., 71:379-406, 1992.

26. E. Artioli, L. Beirão da Veiga, H. Hakula, and C. Lovadina. Free vibrations for some Koiter shells of revolution. Appl. Math. Lett., 21:1245-1248, 2008.

27. R. M. Rosenberg. The normal modes of nonlinear n-degree-of-freedom systems. Journal of Applied Mechanics, 29:7-14, 1962.

28. R. M. Rosenberg. On non-linear vibrations of systems with many degrees of freedom. Advances in Applied Mechanics, 9:155-242, 1966.

29. A. F. Vakakis, L. I. Manevitch, Y. V. Mikhlin, V. N. Philipchuck, and A. A. Zevin. Normal modes and localization in non-linear systems. Wiley, New-York, 1996.

30. A.F. Vakakis. Non-linear normal modes (nnms) and their application in vibration theory: an overview. Mechanical Systems and Signal Processing, 11(1):3-22, 1997.

31. A.F. Vakakis, O.V. Gendelman, L.A. Bergman, D.M. McFarland, G. Kerschen, and Y.S. Lee. Nonlinear targeted energy transfer in mechanical and structural systems I. Springer, New-York, 2008.

32. G. Kerschen, M. Peeters, J.C. Golinval, and A.F. Vakakis. Non-linear normal modes, part I: a useful framework for the structural dynamicist. Mechanical Systems and Signal Processing, 23(1):170-194, 2009.

33. E.J. Doedel, R. Paffenroth, A.R. Champneys, T.F. Fairgrieve, Y.A. Kuznetsov, B.E. Oldeman, B. Sandstede, and X. Wang. Auto 2000: Continuation and bifurcation software for ordinary differential equations. Technical report, Concordia University, 2002. available at http://cmvl.cs.concordia.ca/auto/.

34. C. Touzé, O. Thomas, and M. Amabili. Transition to chaotic vibrations for harmonically forced perfect and imperfect circular plates. International Journal of Non-linear Mechanics, 46(1):234-246, 2011.

35. C. Touzé, S. Bilbao, and O. Cadot. Transition scenario to turbulence in thin vibrating plates. Journal of Sound and Vibration, 331(2):412-433, 2012.

36. M. Ducceschi, C. Touzé, S. Bilbao, and C.J. Webb. Nonlinear dynamics of rectangular plates: investigation of modal interaction in free and forced vibrations. Acta Mechanica, in press, 2013

37. M. Peeters, R. Viguié, G. Sérandour, G. Kerschen, and J.C. Golinval. Non-linear normal modes, part II: toward a practical computation using numerical continuation techniques. Mechanical Systems and Signal Processing, 23(1):195-216, 2009.

38. M. Peeters, G. Kerschen, J.C. Golinval, C. Stephan, and P. Lubrina. Nonlinear normal modes of a full-scale aircraft. In 29th International Modal Analysis Conference, Jacksonville (USA), 2011.

39. F. Blanc, C. Touzé, J.-F. Mercier, K. Ege, and A.-S. Bonnet-Ben-Dhia. On the numerical computation of nonlinear normal modes for reduced-order modelling of conservative vibratory systems. Mechanical Systems and Signal Processing, 36(2):520-539, 2013.

40. E. Hairer, C. Lubich, and G. Wanner. Geometric numerical integration: structurepreserving algorithms for Ordinary differential equations. Springer, 2006. second edition.

41. A. H. Nayfeh and W. Lacarbonara. On the discretization of distributed-parameter systems with quadratic and cubic non-linearities. Nonlinear Dynamics, 13:203-220, 1997.

42. G. Rega, W. Lacarbonara, and A. H. Nayfeh. Reduction methods for nonlinear vibrations of spatially continuous systems with initial curvature. Solid Mechanics and its applications, 77:235-246, 2000.

43. M. Amabili, F. Pellicano, and M. P. Païdoussis. Non-linear dynamics and stability of circular cylindrical shells containing flowing fluid, part III: truncation effect without flow and experiments. Journal of Sound and Vibration, 237(4):617-640, 2000.

44. C. Touzé, O. Thomas, and A. Chaigne. Hardening/softening behaviour in non-linear oscillations of structural systems using non-linear normal modes. Journal of Sound and Vibration, 273(1-2):77-101, 2004.

45. A. H. Nayfeh, J. F. Nayfeh, and D. T. Mook. On methods for continuous systems with quadratic and cubic nonlinearities. Nonlinear Dynamics, 3:145-162, 1992. 
46. M. Pakdemirli, S.A. Nayfeh, and A.H. Nayfeh. Analysis of one-to-one autoparametric resonances in cables. discretization vs. direct treatment. Nonlinear Dynamics, 8:65-83, 1995.

47. M. Amabili. Non-linear vibrations of doubly-curved shallow shells. International Journal of Non-linear Mechanics, 40(5):683-710, 2005.

48. C. Touzé and O. Thomas. Non-linear behaviour of free-edge shallow spherical shells: effect of the geometry. International Journal of Non-linear Mechanics, 41(5):678-692, 2006.

49. S. W. Shaw and C. Pierre. Non-linear normal modes and invariant manifolds. Journal of Sound and Vibration, 150(1):170-173, 1991.

50. C. Touzé and M. Amabili. Non-linear normal modes for damped geometrically nonlinear systems: application to reduced-order modeling of harmonically forced structures. Journal of Sound and Vibration, 298(4-5):958-981, 2006. 